\begin{tabular}{|c|c|}
\hline \multicolumn{2}{|c|}{ Access this article online } \\
\hline Www.klinikaoczna.pl \\
\hline
\end{tabular}

\title{
Ophthalmic contraindications to sports
}

\author{
Marek R. Szaliński', Artur Pupka ${ }^{2,3}$, Marta Misiuk-Hojło ${ }^{1}$ \\ ${ }^{1}$ Chair and Department of Ophthalmology, Wroclaw Medical University, Poland \\ ${ }^{2}$ Center for the Treatment of Vascular Diseases and Sports Medicine, Wroclaw, Poland \\ ${ }^{3}$ Doctor of the National Team of the Polish Triathlon Union
}

\begin{abstract}
Sports activity in ophthalmic patients is an insufficiently understood and developed topic, which is reflected in the small number of publications on this subject and the lack of precise guidelines available both in the materials of scientific societies and in textbooks used by doctors during preparation for the specialization exam. The number of amateur sports people has been growing recently and questions about the possibility of undertaking physi-
\end{abstract}

cal effort after surgery are common among ophthalmic patients. The authors of the article review the available sources of knowledge to bring closer the issues indicated in the title of the work. Conditions after glaucoma and retinal detachment, refractive operations and other inoperable conditions such as central serous retinopathy and eye surface diseases are discussed.

KEY WORDS: sport, ophthalmology, postoperative recomendations.
In view of the growing popularity of sporting activities, especially among adults but also in the age group associated with the development of chronic diseases, there is an ever urgent need to adequately address the risk factors and medical contraindications to involvement in sports. This applies in particular to sporting disciplines which require extreme physical effort, such as marathon or triathlon and martial arts. The latter, in their contact options, are mainly practiced by individuals between 15 and 40 years of age [1].

Patients with some ophthalmic diseases need to face certain limitations associated with the practice of sports - or rather the choice of appropriate disciplines. Especially affected are individuals after laser-assisted corneal refractive surgery and other surgical procedures, patients with ocular surface autoimmune diseases, glaucoma with various pathogenic mechanisms, lens abnormalities, retinal diseases (mainly retinal detachment), and asymptomatic peripheral retinal degenerations [2].

\section{PATIENTS AFTER LASER-ASSISTED CORNEAL REFRACTIVE SURGERY}

Laser-assisted corneal refractive surgery is a fairly common method of correcting vision defects (myopia, astigmatism and hyperopia), and generally recommended as the method of choice in sports-practicing individuals. However, in cases where eye injury may occur, for example in combat sports or team games (volleyball, hockey) LASIK (laser-assisted in situ keratomileusis) is a technique to be avoided because despite the lack of post-surgical discomfort, it involves the creation a corneal flap which never fully heals. As a result, there is a risk of flap dislodgement or detachment - and thus permanent deterioration of vision - following eye injury in which the force acts tangentially to the ocular surface $[3,4]$. Consequently, superficial laser-assisted vision correction methods should definitely be selected in the treatment of patients who want to practice various sports. Such methods include LASEK (laser subepithelial keratomileusis) or epi-LASIK (epithelial laser in situ keratomileusis), where no flap is created. The procedures are associated with several days of discomfort for the patients.

Participation in no-contact sports, such as running, skiing or cycling, is advisable. Swimming can be resumed relatively quickly, but always after complete corneal healing. Typically, patients are advised to refrain from sports for 2-4 weeks after the procedure. The latter (longer) period should be maintained in individuals who want to use water slides in public water amusement parks. If swimming training cannot be interrupted, an intracorneal technique for vision correction (SMILE) may be recommended.

\section{OCULAR SURFACE AUTOIMMUNE DISEASES}

The most common autoimmune diseases which prompt patients to make an appointment with an ophthalmologist in- 
clude rheumatoid arthritis and inflammations of small blood vessels, presenting as chronic sterile ulceration and thinning of the cornea, occasionally leading to corneal perforation [5]. The patient's age and condition may be factors preventing the practice of extreme sports, and caution should be exercised when swimming, regardless of water body type. The loss of corneal epithelium may be conducive to corneal infections with bacteria, fungi and ameba which can quickly lead to the destruction of the cornea and possibly loss of the eye. Autoimmune diseases may have different degrees of severity. Occasionally, they run a subclinical course. If such conditions are not associated with ocular surface abnormalities, water sports are not contraindicated. In fact, patients with rheumatoid arthritis are often advised to take up swimming as a form of rehabilitation. In ophthalmic disorders of minor severity, such as peripheral ingrowth of blood vessels, peripheral corneal thinning or dry eye syndrome, patients are free to engage in jogging, cycling or skiing at the amateur level, but appropriate eyewear and even protective eye goggles are recommended, as is the application of preservative-free artificial tears because of excessive tear film evaporation associated with these sports [6].

\section{GLAUCOMA}

Glaucoma is a disease associated with the destruction of retinal ganglion cells in the mechanism of apoptosis, and leads to permanent blindness. Glaucoma may develop through various pathogenetic mechanisms, which makes it a very heterogeneous disease. Consequently, contraindications to sports activities depend on the type of glaucoma and the degree of visual field loss [7].

Pigmentary glaucoma affects mostly men between 20 and 40 years of age, and involves excessive pigment release from the posterior iris while rubbing against the ciliary zonule and the lens [8]. The released pigment clogs the drainage angle, triggering an intraocular pressure surge and slowly progressive damage to the optic nerve. Patients may report visual disturbances manifesting as rainbows around light sources associated with transient corneal edema. Laser treatment reduces the problem of pigment release, but does not significantly affect the issue of sports practice, as abrupt head movements and extreme physical effort continue to be contraindicated. The only permanent resolution of the problem is surgical treatment. Bilateral trabeculectomy makes the level of intraocular pressure independent of the presence of pigment in the anterior chamber, thus permitting any type of sporting activity. An example is one of the Dutch footballers who, after bilateral trabeculectomy, wore protective goggles over a prolonged period of time while playing football in order to prevent eye injury.

Protective eye goggles or a plastic mask provide a safeguard against injuries, so they seem a necessary method of protection in patients after intraocular surgery [9].

Primary open-angle glaucoma is the most common type of glaucoma, developing later in life, after 40 years of age, but more typically in individuals older than 60 years.
The pathomechanism of the condition is not completely understood, but the effects of hypoxia and ischemia on the optic disc and retina have long been postulated as contributory factors. In patients with the condition, playing sports is strongly recommended, but in my own practice, I once had a patient whose visual field deteriorated over the course of several years, probably as a result of running marathons $[10$, 11]. Such extreme physical effort may be associated with metabolic disorders and oxygen deficiency, which may also adversely affect the supply of oxygen to the retinal ganglion cells. There are reports showing that marathon-related physical exercise has no effect on retinal blood flow parameters. However, the reports relate to the general population, without specifically considering patients with advanced glaucoma whose microcirculatory autoregulation mechanism may be considerably different from normal [12].

It needs to be stressed that running is generally seen as a factor which prevents the development of primary openangle glaucoma [13], and contributes to a decrease in intraocular pressure $[14,15]$. Sports such as swimming, cycling or skiing, practiced at the amateur level, should also be considered as beneficial for the patient.

Other forms of glaucoma are not regarded as significant contraindications to sports, except when advanced visual field defects have occurred [7]. The reason may be that patients with peripheral visual field defects are prone to injuries when practicing sports involving fast movements, such as alpine skiing, hockey or football, which require good peripheral vision. Tennis, table tennis or generally individual sports seem to be more appropriate in such cases, as the central visual field is primarily involved in the sporting competitive process.

\section{MISALIGNMENT OF THE LENS}

Upward lens subluxation is a characteristic feature of Marfan syndrome, and represents a contraindication to practicing combat sports. It should be noted, though, that Marfan syndrome has a large variability of phenotypes. Often, the disease causes no major deviations from the norm, and does not preclude participation in sports even at a very high level of competition, especially basketball [16]. Sports which do not require abdominal muscle activation, such as cycling and swimming, can be beneficial to the patient. However, running - especially for long distances - is a controversial topic. Patients who want to exercise in the gym should first be assessed to determine their cardiovascular health, focusing on the heart and thoracic aorta, because of potential risks involved in the Valsalva maneuver, i.e. contraction of the abdominal muscles against a closed glottis. Generally, strenuous exercise - involving both static and dynamic activity - is contraindicated in all types of sports in view of the risk of aortic dissecting aneurysm and sudden death [17]. Patients should avoid weight training or gym exercises with heavy loads.

\section{CENTRAL SEROUS RETINOPATHY}

The etiology of CSR is probably associated with impaired blood supply to the choroid capillary layer and functional dis- 
orders of the retinal pigment epithelium. It is characterized by a minor defect in the central visual field which, however, causes the patient considerable discomfort. In the acute phase of the disease, in which stress is a contributory factor, potentially along with excessive activity of the sympathetic autonomic nervous system and endogenous corticosteroids, physical exercise, especially of extreme severity, is contraindicated $[18,19]$. In the chronic or recurrent phase, diving should be avoided because of episodes of retinal and choroidal perfusion disorders associated with decompression processes that accompany surfacing from the depth of over 30-40 meters [20].

Patients with a history of CRS episodes should not stay away from physical exercise - quite the opposite. In fact, regular sporting activity helps to relieve stress, which is a risk factor $[21,22]$. However, patients who practice body-building should be advised not to take exogenous anabolic steroids. Extreme effort can also be viewed as controversial.

\section{RETINAL DETACHMENT}

Patients with lattice degenerations in the peripheral retina, and after surgical treatment of retinal detachment using the method of scleral buckling, should avoid abrupt head movements. The reason is that vibrations of the vitreous body may cause retinal detachment. As a result, patients are advised against contact sports, such as judo or basketball [23]. It is also debatable whether running is advisable. In contrast, cycling and swimming are strongly recommended.

However, no significant contraindications exist after vitrectomy, i.e. another type of retinal surgery, involving the removal of the vitreous body. The use of protective eye goggles should be considered $[6,9]$.

It is worth noting that boxing and basketball are the main sports disciplines associated with retinal detachment [24].

In conclusion, it is also important to highlight the favorable effects of sports activities on health, especially long-term benefits on the cardiovascular system [25]. For this reason, in the treatment of patients with ophthalmic diseases, physicians should not focus on the general contraindications to engaging in sports, but instead try to suggest sports disciplines that will be both attractive and safe for the patient.

\section{AFTER VITRECTOMY OR CATARACT SURGERY}

Some of the frequent reasons for ophthalmic consultations nowadays are follow-up evaluations after cataract surgery or vitrectomy. Typically, patients want to find out what types of exercise or physical activity are contraindicated, and what limitations are associated with surgery.

Following cataract surgery performed by the standard procedure, where the ciliary zonule is undamaged and the IOL is placed inside the capsular bag, physical exercise and participation in sports should definitely be restricted during the early postoperative period. During the first 7 days, eye drops are administered at relatively the highest frequency, so in this period the postoperative wounds may not yet be completely closed [26]. Under compression, they may easily open, leading to intraocular infection. As a consequence, physical activity is not recommended during this period. Between the postoperative days 7 and 30, competitive sports and disciplines associated with a risk of eye injuries, such as basketball, football, and combat sports, should be avoided. After a month, the corneal wounds are closed and the IOL adheres to the capsular bag relatively well (especially if it is highly hydrophobic) [27]. From that point on, patients have essentially no contraindications to engage in any sports.

Pars plana vitrectomy is a procedure performed in a wide variety of indications. Consequently, even among patients with the same disease, the surgical and postoperative course can be highly variable. Generally, patients should spend the first month after the procedure resting, and avoiding sudden head movements. Practicing sports - except for disciplines such as golf, bowling or table tennis (though not at the professional level) - is contraindicated. After approximately 2 months, the adhesion between the retina, retinal pigment epithelium and choroid is improved at the sites of laser therapy and cryotherapy. Also, a relative degree of certainty is reached after all vitreoretinal procedures that the recurrence of retinal detachment is unlikely over a prolonged postoperative period [28, 29]. Between 2 and 3 months after vitrectomy, patients are free to resume even extreme sports, but they should be informed about the risk of retinal detachment and the need to self-monitor and immediately report to the ophthalmic emergency department if they notice a "veil" in the field of vision. Practicing sports at the amateur level - be it running, cycling or ball games - is not expected to increase the risk of late complications after a longer period from surgery.

Following silicone oil endotamponade, until the oil is removed, engaging in sports activities is, in fact, safer for the patients than under normal circumstances.

\section{DISCLOSURE}

The authors declare no conflict of interest.

\section{References}

1. Number of participants in triathlons in the United States from 2006 to 2017 (in millions). Available at: https://www.statista.com/ statistics/191339/participants-in-triathlons-in-the-us-since-2006/.

2. Micieli JA, Easterbrook M. Eye and orbital injuries in sports. Clin Sports Med 2017; 36: 299-314.

3. Tetz M, Werner L, Muller M, Dietze U. Late traumatic LASIK flap loss during contact sport. J Cataract Refract Surg 2007; 33: 13321335.

4. Aldave AJ, Hollander DA, Abbott RL. Late-onset traumatic flap dislocation and diffuse lamellar inflammation after laser in situ keratomileusis. Cornea 2002; 21: 604-607.

5. Cao Y, Zhang W, Wu J, et al. Peripheral ulcerative keratitis associated with autoimmune disease: pathogenesis and treatment. J Ophthalmol 2017; 2017: 7298026. 
6. Erickson GB. Sports Vision. Chapter 6. Butterworth Heinemann. Elsevier. St. Louis. Missouri 2007.

7. Ramulu P. Glaucoma and disability: which tasks are affected, and at what stage of disease? Curr Opin Ophthalmol 2009; 20: 92-98.

8. Haynes WL, Johnson AT, Alward WL. Effects of jogging exercise on patients with the pigmentary dispersion syndrome and pigmentary glaucoma. Ophthalmology 1992; 99: 1096-1103.

9. Micieli JA, Zurakowski D, Ahmed IIK. Can J Ophthalmol 2014; 49: 243-248.

10. Khan H, Phillipose J, Ahmed M, Deeb L. Athlete's Hepatitis in a Young Healthy Marathon Runner. Case Rep Gastroenterol 2018; 12: 176-181.

11. Iqbal FM, Goparaju M, Yemme S, Lewis BE. Renal artery dissection following marathon running. Angiology 2009; 60: 122-126.

12. Pressler A, Hanssen $\mathrm{H}$, Dimitrova $\mathrm{M}$, et al. Acute and chronic effects of marathon running on the retinal microcirculation. Atherosclerosis 2011; 219: 864-868.

13. Williams PT. Relationship of incident glaucoma versus physical activity and fitness in male runners. Med Sci Sports Exerc 2009; 41: 1566-1572.

14. Bozkurt $B, 0$ okudan $N$, Belviranli $M$, Oflaz AB. The evaluation of intraocular pressure fluctuation in glaucoma subjects during submaximal exercise using an ocular telemetry sensor. Indian J Ophthalmol 2019; 67: 89-94.

15. Roddy G, Curnier D, Ellemberg D. Reductions in intraocular pressure after acute aerobic exercise: a meta-analysis. Clin I Sport Med 2014; $24: 364-372$.

16. Braverman AC. Recognizing Marfan syndrome in athletes. Dostępne na: https://www.acc.org/latest-in-cardiology/articles/2015/03/02/13/03/recognizing-marfan-syndrome-in-athletes.

17. Braverman AC. Exercise and the Marfan syndrome. Med Sci Sport Exerc 1998; (10 Suppl): 387-395.

18. Daruich A, Matet A, Dirani A, et al. Central serous chorioretinopathy: recent findings and new physiopathology hypothesis. Prog Retin Eye Res 2015; 48: 82-118.

19. Haimovici R, Koh S, Gagnon DR, et al. Central Serous Chorioretinopathy Case-Control Study Group. Risk factors for central serous chorioretinopathy: a case-control study. Ophthalmology 2004; 111:244-249.

20. Iordanidou V, Gendron G, Khammari C, et al. Choroidal ischemia secondary to a diving injury. Retin Cases Brief Rep 2010; 4: 262-265.

21. Bazzazi N, Ahmadpanah M, Akbarzadeh S, et al. In patients suffering from idiopathic central serous chorioretinopathy, anxiety scores are higher than in healthy controls, but do not vary according to sex or repeated central serous chorioretinopathy. Neuropsychiatr Dis Treat 2015; 11: 1131-1136.

22. Hansmann R, Hug SM, Seeland K. Restoration and stress relief through physical activities in forests and parks. Urban Forestry and Urban Greening, 2007; 6: 213-225.

23. Lee TH, Chen YH, Kuo HK, et al. Retinal detachment associated with basketball-related eye trauma. Am J Ophthalmol 2017; 180: 97-101.

24. Karpman S, Reid P, Phillips L, et al. Combative Sports Injuries: An Edmonton Retrospective. Clin J Sport Med 2016; 26: 332-334.

25. Lundberg K, Tarp J, Vestergaard AH, et al. Retinal vascular diameters in relation to physical activity in Danish Children - The CHAMPS Eye Study. Scand J Med Sci Sports 2018; 28: 1897-1907.

26. Matossian C, Makari S, Potvin R. Catarct surgery ad methods of wound closure: a review. Clin Ophthalmol 2015; 9: 921-928.

27. Zhu X, He W, Yang J, et al. Adhesion of the posterior capsule to different intraocular lenses following cataract surgery. Acta Ophthalmol 2016; 94: e16-25.

28. Wilkinson JT, Richards $A B$, Choi D, et al. Incidence of retinal detachment after fellow-performed primary pars plana vitrectomy. ISRN Ophthalmol 2013; 2013: 353209.

29. Mietz H, Heimann K. Onset and reccurence of prolifarative vitreoretinopathy in various vitreoretinal diseases. Br J Ophthalmol 1995; 79: 874-877. 